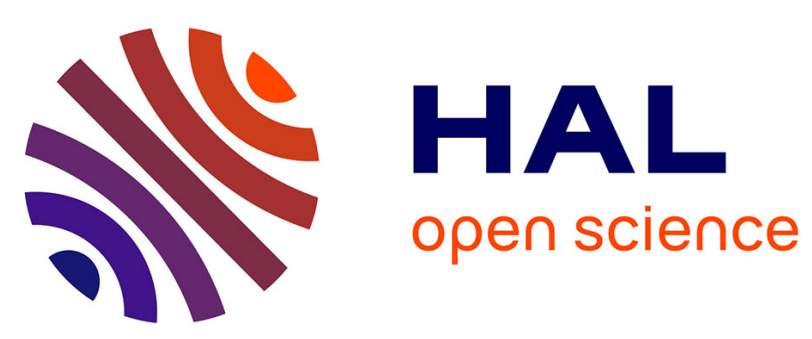

\title{
Exact solution of a 1D asymmetric exclusion model using a matrix formulation
}

\author{
Bernard Derrida, M. R. Evans, V. Hakim, V. Pasquier
}

\section{To cite this version:}

Bernard Derrida, M. R. Evans, V. Hakim, V. Pasquier. Exact solution of a 1D asymmetric exclusion model using a matrix formulation. Journal of Physics A: Mathematical and General (1975 - 2006), 1993, 26 (7), pp.1493-1517. 10.1088/0305-4470/26/7/011 . hal-03282962

\section{HAL Id: hal-03282962 \\ https://hal.science/hal-03282962}

Submitted on 19 Jul 2021

HAL is a multi-disciplinary open access archive for the deposit and dissemination of scientific research documents, whether they are published or not. The documents may come from teaching and research institutions in France or abroad, or from public or private research centers.
L'archive ouverte pluridisciplinaire HAL, est destinée au dépôt et à la diffusion de documents scientifiques de niveau recherche, publiés ou non, émanant des établissements d'enseignement et de recherche français ou étrangers, des laboratoires publics ou privés. 


\title{
Exact solution of a $1 \mathrm{D}$ asymmetric exclusion model using a matrix formulation
}

\author{
B Derrida†, M R Evans $\nmid, \mathrm{V}$ Hakim $\ddagger$ and V Pasquier $\dagger$ \\ † Service de Physique Théorique, Laboratoire de la Direction des Sciences de ta Matière \\ du Commissariat à l'Energie Atomique, CE Saclay, F-91191 Gif-sur-Yvette Cedex, \\ France \\ ¥ Laboratoire de Physique Statistique de l'Ecole Normale Supérieure, Laboratoire \\ associé au Centre National de la Recherche Scientifique, 24 rue Lhomond, F-75231 Paris \\ Cedex 05, France
}

Received 30 November 1992

\begin{abstract}
Several recent works have shown that the one-dimensional fully asymmetric exclusion model, which describes a system of particles hopping in a preferred direction with hard core interactions, can be solved exactly in the case of open boundaries. Here we present a new approach based on representing the weights of each configuration in the steady state as a product of non-commuting matrices. With this approach the whole solution of the problem is reduced to finding two matrices and two vectors which satisfy very simple algebraic rules. We obtain several explicit forms for these non-commuting matrices which are, in the general case, infinite-dimensional. Our approach allows exact expressions to be derived for the current and density profiles. Finally we discuss briefly two possible generalizations of our results: the problem of partially asymmetric exclusion and the case of a mixture of two kinds of particles.
\end{abstract}

\section{Introduction}

Systems of interacting particles with stochastic dynamics have been studied for quite some time both in the mathematical and physical literature (Spitzer 1970, Liggett 1985, Spohn 1991). The main mathematical achievements have been to allow an understanding of the asymptotic measures (Liggett 1975, 1977, Andjel et al 1988), the fluctuations of tagged particles (Kipnis 1986, Ferrari 1986) and the microscopic structure of shocks (De Masi et al 1988, Bramson 1988, Ferrari et al 1991, Ferrari 1992). From a physical viewpoint, driven lattice gases with hard core repulsion provide models for the diffusion of particles through narrow pores and for hopping conductivity (Richards 1977, Katz et al 1984), and belong to the general class of non-equilibrium models which includes driven diffusive systems (van Beijeiren et al 1983, Kipnis et al 1983). They are closely linked to growth processes (Meakin et al 1986, Dhar 1987, Krug and Spohn 1991, Kandel and Mukamel 1992) and can also be formulated as traffic jam or queuing problems (Kipnis 1986, Jean-Marie and Massey 1992).

The asymmetric exclusion model in one dimension is one of the simplest examples of a driven diffusive system (Liggett 1985, Andjel et al 1988, Bramson 1988, De Masi et al 1988, Ferrari et al 1991, Ferrari 1992, Gwa and Spohn 1992, Janowsky and 
Lebowitz 1992). Here we study the version with open boundary conditions. The model comprises particles which jump independently to their right with hard core repulsion along a one-dimensional lattice, open boundary conditions meaning that particles are injected at one end of the lattice and are removed at the opposite end. It has been noticed that the steady state of the system can be obtained exactly as a simple recursion relation on the system size (Derrida et al 1992, Liggett 1975, JeanMarie and Massey 1992). This recursion was used to calculate exactly, in the steady state, expressions of the density profile (the one-point correlation function) (Derrida et al 1992) and for higher correlation functions (Derrida and Evans 1993). In these works the route from the recursion relation to the exact expression of the above quantities was via a rather complicated generating function method.

In the present paper we describe a different approach which is inspired by techniques used in the study of integrable systems (Faddeev 1980, Baxter 1982). It consists of representing the weights of the configurations in the steady state as a product of non-commuting matrices. The technique has previously been applied to the problems of directed lattice animais (Hakim and Nadal 1983) and quantum antiferromagnetic spin chains (Klümper et al 1991, Fannes et al 1992). For the asymmetric exclusion process the present approach simplifies the derivation of previous results (Derrida et al 1992, Derrida and Evans 1993) and facilitates their generalization.

The paper is organized as follows. In section 2 we recall the definition of the model we consider and explain how one can represent the weights of the configurations in the steady state as products of non-commuting matrices. In section 3 , we prove that this matrix formulation indeed gives the steady state. In section 4 , we give explicit forms of the matrices and in section 5 we present exact expressions of the density profiles for finite systems. In the next four sections we then proceed to examine the limit of infinite systems. In section 6 we obtain several asymptotic forms for matrix elements which are needed in the calculation of the density profiles. The current is then computed in section 7, giving rise to a phase diagram which consists of three phases: a low-density phase, a high-density phase and a maximal current phase. In section 8 , we compute the density far from the boundaries and in section 9 , we find the density profiles near the boundaries. In section 10 , we show that previously known results in a special case (Derrida et al 1992, Derrida and Evans 1993) can be recovered very easily with the matrix approach. Finally, in section 11 we suggest two possible generalizations of the problem which may be tackled by similar matrix approaches.

\section{Definition of the model and steady state}

Let us first define the dynamics of the one-dimensional asymmetric exclusion model with open boundary conditions that we consider here (Derrida et al 1992). Each site $i(1 \leqslant i \leqslant N)$ of a one-dimensional lattice of $N$ sites is either occupied by a particle $\left(\tau_{i}=1\right)$ or empty $\left(\tau_{i}=0\right)$. During every infinitesimal time interval $\mathrm{d} t$, each particle in the system has a probability $\mathrm{d} t$ of jumping to the next site on its right (for all particles on sites $1 \leqslant i \leqslant N-1$ ) if this neighbouring site is empty. Furthermore, a particle is added at site $i=1$ with probability $\alpha \mathrm{d} t$ if site 1 is empty and a particle is removed from site $N$ with probability $\beta \mathrm{d} t$ if this site is occupied. Thus if the system is in configuration $\left\{\tau_{1}, \tau_{2}, \ldots, \tau_{N}\right\}$ at time $t$, its configuration becomes at time $t+\mathrm{d} t$ 
for $i=1$

$$
\begin{aligned}
& \tau_{1}(t+\mathrm{d} t)=1 \quad \text { with probability } \quad p_{1}=\tau_{1}+\left[\alpha\left(1-\tau_{1}\right)-\tau_{1}\left(1-\tau_{2}\right)\right] \mathrm{d} t \\
& =0 \quad \text { with probability } \quad 1-p_{1}
\end{aligned}
$$

for $2 \leqslant i \leqslant N-1$

$$
\begin{aligned}
& \tau_{i}(t+\mathrm{d} t)=1 \quad \text { with probability } \quad p_{i}=\tau_{i}+\left[\tau_{i-1}\left(1-\tau_{i}\right)-\tau_{i}\left(1-\tau_{i+1}\right)\right] \mathrm{d} t \\
& =0 \quad \text { with probability } \quad 1-p_{i}
\end{aligned}
$$

for $i=N$

$$
\begin{aligned}
& \tau_{N}(t+\mathrm{d} t)=1 \quad \text { with probability } \quad p_{N}=\tau_{N}+\left[\tau_{N-1}\left(1-\tau_{N}\right)-\beta \tau_{N}\right] \mathrm{d} t \\
& =0 \quad \text { with probability } \quad 1-p_{N}
\end{aligned}
$$

In the long time limit, the system reaches a steady state where all the probabilities $P_{N}\left(\tau_{1}, \tau_{2}, \ldots, \tau_{N}\right)$ of finding the system in configurations $\left\{\tau_{1}, \tau_{2}, \ldots, \tau_{N}\right\}$ are stationary, i.e. satisfy

$$
\frac{\mathrm{d}}{\mathrm{d} t} P_{N}\left(\tau_{1}, \tau_{2}, \ldots, \tau_{N}\right)=0
$$

Our approach allows one to obtain exact expressions for all the $P_{N}\left(\tau_{1}, \tau_{2}, \cdots \tau_{N}\right)$. As already mentioned in previous works (Derrida et al 1992, Derrida and Evans 1993), it is more convenient to consider unnormalized weights $f_{N}\left(\tau_{1}, \tau_{2}, \ldots, \tau_{N}\right)$ which are equal to the $P_{N}\left(\tau_{1}, \tau_{2}, \ldots, \tau_{N}\right)$ up to a multiplicative constant (independent of the configuration):

$$
P_{N}\left(\tau_{1}, \tau_{2}, \ldots, \tau_{N}\right)=f_{N}\left(\tau_{1}, \tau_{2}, \ldots, \tau_{N}\right) / Z_{N}
$$

with

$$
Z_{N}=\sum_{\tau_{1}=0,1} \cdots \sum_{\tau_{N}=0,1} f_{N}\left(\tau_{1}, \tau_{2}, \ldots, \tau_{N}\right) .
$$

By analogy with other exactly soluble models (Hakim and Nadal 1983, Klümper et al 1991, Fannes et al 1992) we shall show in the next section that the $f_{N}\left(\tau_{1}, \tau_{2}, \ldots, \tau_{N}\right)$ in the steady state can be obtained from the following expression:

$$
f_{N}\left(\tau_{1}, \tau_{2}, \ldots, \tau_{N}\right)=\left\langle W\left|\prod_{i=1}^{N}\left(\tau_{i} D+\left(1-\tau_{i}\right) E\right)\right| V\right\rangle
$$

where $D$ and $E$ are square matrices and $|V\rangle$ and $\langle W|$ are vectors satisfying

$$
\begin{aligned}
& D E=D+E \\
& D|V\rangle=\frac{1}{\beta}|V\rangle \\
& \langle W| E=\frac{1}{\alpha}\langle W| .
\end{aligned}
$$


Notation (7) simply means that the weight $f_{N}\left(\tau_{1}, \tau_{2}, \ldots, \tau_{N}\right)$ is given by a product of $N$ matrices $D$ or $E$ with matrix $D$ at position $i$ if site $i$ is occupied $\left(\tau_{i}=1\right)$ and matrix $E$ if site $i$ is empty $\left(\tau_{i}=0\right)$. We stress here that equations (7)-(10) are the essence of our approach; all results of sections 5-10 are direct consequences thereof.

Before proving that the conditions (8)-(10) indeed give the steady state and before presenting explicit forms for the matrices and vectors involved, let us discuss the advantages of the approach. If one defines the matrix $C$ by

$$
C=D+E
$$

it is clear that $\left\langle\tau_{i}\right\rangle_{N}$ defined by

$$
\left\langle\tau_{i}\right\rangle_{N}=\sum_{\tau_{1}=1,0} \ldots \sum_{\tau_{N}=1,0} \tau_{i} f_{N}\left(\tau_{1}, \ldots, \tau_{N}\right)\left[\sum_{\tau_{1}=1,0} \ldots \sum_{\tau_{N}=1,0} f_{N}\left(\tau_{1}, \ldots, \tau_{N}\right)\right]^{-1}
$$

can be calculated through the following formula

$$
\left\langle\tau_{i}\right\rangle_{N}=\frac{\left\langle W\left|C^{i-1} D C^{N-i}\right| V\right\rangle}{\left\langle W\left|C^{N}\right| V\right\rangle} .
$$

Similarly, higher correlation functions will have simple expressions in terms of these matrices. For example, when $i<j,\left\langle\tau_{i} \tau_{j}\right\rangle_{N}$,

$$
\left\langle\tau_{i} \tau_{j}\right\rangle_{N}=\frac{\left\langle W\left|C^{i-1} D C^{j-i-1} D C^{N-j}\right| V\right\rangle}{\left\langle W\left|C^{N}\right| V\right\rangle} .
$$

Therefore, if we have convenient forms for the matrices $D, E$ and the vectors $\langle W|| V$,$\rangle so that matrix elements of any power of C$ have simple expressions, then formulae for the density profile $\left\langle\tau_{i}\right\rangle_{N}$ and for higher correlations $\left\langle\tau_{i} \ldots \tau_{j}\right\rangle_{N}$ will follow easily.

Remark. In the steady state, one can derive a hierarchy of relations between the correlation functions from the conditions

$$
\frac{\mathrm{d}}{\mathrm{d} t}\left\langle\tau_{i_{1}} \tau_{i_{2}} \cdots \tau_{i_{k}}\right\rangle=0
$$

For example, writing that

$$
\frac{\mathrm{d}}{\mathrm{d} t}\left\langle\tau_{i}\right\rangle_{N}=0
$$

for $1 \leqslant i \leqslant N$ implies

$$
\alpha\left\langle W\left|E C^{N-1}\right| V\right\rangle=\cdots=\left\langle W\left|C^{i-1} D E C^{N-i-1}\right| V\right\rangle=\cdots=\beta\left\langle W\left|C^{N-1} D\right| V\right\rangle .
$$

These relations (17) can be understood very easily in terms of the conservation of the current of particles. Each equality expresses the fact that the current between site $i$ and site $i+1$ is independent of $i$ for $0 \leqslant i \leqslant N$. Clearly, all these relations are satisfied immediately when $D, E,|V\rangle$ and $\langle W|$ obey (8)-(11).

Remark. We showed in a preliminary version of this work (Derrida et al 1993) that when the $f_{N}\left(\tau_{1}, \cdots, \tau_{N}\right)$ are given by the expressions (7)-(10), they satisfy the recursions which were derived in Derrida et al (1992 equation (8)) and also used in Derrida and Evans (1993). Similar recursions were proved some time ago by Liggett (1975). 


\section{Proof of stationarity}

In this section we shall prove that (7), with $D, E,\langle W|$ and $|V\rangle$ chosen to satisfy the algebra $(8)-(10)$, does indeed give the weights of the configurations in the steady state. The proof we present here is an alternative to using the recursion relation on the system size for the steady state, on which a previous approach to the problem was built (Derrida et al 1992). Our method of proof is closely related to an argument of Sutherland (1970).

Let us begin by writing down matrices that represent the transition rates into configurations (off-diagonal elements) and out of configurations (diagonal elements). We first construct a matrix $h_{1}$ that represents transition rates due to particles entering at the left-hand boundary site. The rate at which particles attempt to enter, $\alpha$, gives both $\left(h_{1}\right)_{1 ; 0}$ (the transition rate into $\left(1, \tau_{2}, \ldots, \tau_{N}\right)$ from configuration $\left(0, \tau_{2}, \ldots, \tau_{N}\right)$ ) and $-\left(h_{1}\right)_{0 ; 0}$ (the transition rate out of $\left.\left(0, \tau_{2}, \ldots, \tau_{N}\right)\right)$. Since a particle cannot leave the system at the left boundary, $\left(h_{1}\right)_{0,1}$ (the transition rate into $\left(0, \tau_{2}, \ldots, \tau_{N}\right)$ from $\left(1, \tau_{2}, \ldots, \tau_{N}\right)$ ) and $-\left(h_{1}\right)_{1 ; 1}$ (the transition rate out of $\left.\left(1, \tau_{2}, \ldots, \tau_{N}\right)\right)$ are both zero. We may thus write a matrix $h_{1}$ for the transition rates between the two configurations $\left(0, \tau_{2}, \ldots, \tau_{N}\right),\left(1, \tau_{2}, \ldots, \tau_{N}\right)$ due to particles entering as

$$
h_{1}=\left(\begin{array}{cc}
-\alpha & 0 \\
\alpha & 0
\end{array}\right)
$$

where the basis is $\left\{\left(0, \tau_{2}, \ldots, \tau_{N}\right) ;\left(1, \tau_{2}, \ldots, \tau_{N}\right)\right\}$ or in an obvious shorthand $\{0 ; 1\}$. In a similar fashion we may write a matrix $h_{N}$ representing the transition rates due to particles leaving from the right-hand boundary site

$$
h_{N}=\left(\begin{array}{cc}
0 & \beta \\
0 & -\beta
\end{array}\right)
$$

where the basis is $\{0 ; 1\}$. The transition that occurs due to a particle hopping between a pair of non-boundary sites $i, i+1$ is from $\left(\tau_{1} \cdots \tau_{i-1} 1,0, \tau_{i+2} \cdots \tau_{N}\right)$ to $\left(\tau_{1} \cdots \tau_{i-1} 0,1, \tau_{i+2} \cdots \tau_{N}\right)$. A $4 \times 4$ matrix that represents the transition rates into configurations due to such hops is

$$
h=\left(\begin{array}{cccc}
0 & 0 & 0 & 0 \\
0 & 0 & 1 & 0 \\
0 & 0 & -1 & 0 \\
0 & 0 & 0 & 0
\end{array}\right)
$$

where the basis is $\{0,0 ; 0,1 ; 1,0 ; 1,1\}$. Thus, the only non-zero elements of transition rate matrices are

$$
\begin{array}{ll}
\left(h_{1}\right)_{0 ; 0}=-\alpha & \left(h_{1}\right)_{1 ; 0}=\alpha \\
(h)_{0,1 ; 1,0}=1 & (h)_{1,0 ; 1,0}=-1 \\
\left(h_{N}\right)_{0 ; 1}=\beta & \left(h_{N}\right)_{1 ; 1}=-\beta .
\end{array}
$$

The fact that the elements of the each column of all the matrices (18)-(20) add up to zero reflects the conservation of probability. We now write the evolution equation 
for $P_{N}$ in the following way:

$$
\begin{aligned}
\frac{\mathrm{d}}{\mathrm{d} t} P_{N}\left(\tau_{1}, \tau_{2}, \ldots, \tau_{N}\right)=\sum_{\sigma_{1}}\left(h_{1}\right)_{\tau_{1} ; \sigma_{1}} P_{N}\left(\sigma_{1}, \tau_{2}, \ldots, \tau_{N}\right) \\
\quad+\sum_{i=1}^{N-1} \sum_{\sigma_{i}, \sigma_{i+1}}(h)_{\tau_{1}, \tau_{i+1} ; \sigma_{i}, \sigma_{i+1}} P_{N}\left(\tau_{1}, \ldots, \sigma_{i}, \sigma_{i+1}, \ldots, \tau_{N}\right) \\
+\sum_{\sigma_{N}}\left(h_{N}\right)_{\tau_{N} ; \sigma_{N}} P_{N}\left(\tau_{1}, \ldots, \tau_{N-1}, \sigma_{N}\right) .
\end{aligned}
$$

Let us assume that there exist two coefficients $x_{0}, x_{1}$, such that the following conditions are satisfied for each choice of $\tau_{i}$ :

$$
\begin{aligned}
& \sum_{\sigma_{1}}\left(h_{1}\right)_{\tau_{1} ; \sigma_{1}} P_{N}\left(\sigma_{1}, \tau_{2}, \ldots, \tau_{N}\right)=x_{\tau_{1}} P_{N-1}\left(\tau_{2}, \ldots, \tau_{N}\right) \\
& \sum_{\sigma_{i}, \sigma_{t+1}}(h)_{\tau_{z}, \tau_{t+1} ; \sigma_{t}, \sigma_{t+1}} P_{N}\left(\tau_{1}, \ldots, \sigma_{i}, \sigma_{i+1}, \ldots, \tau_{N}\right) \\
& =-x_{\tau_{i}} P_{N-1}\left(\tau_{1}, \ldots, \tau_{i-1}, \tau_{i+1}, \ldots, \tau_{N}\right) \\
& +x_{\tau_{i+1}} P_{N-1}\left(\tau_{1}, \ldots, \tau_{i}, \tau_{i+2}, \ldots, \tau_{N}\right) \\
& \sum_{\sigma_{N}}\left(h_{N}\right)_{\tau_{N} ; \sigma_{N}} P_{N}\left(\tau_{1}, \ldots, \tau_{N-1}, \sigma_{N}\right)=-x_{\tau_{N}} P_{N-1}\left(\tau_{1}, \ldots, \tau_{N-1}\right)
\end{aligned}
$$

If such coefficients $x_{0}, x_{1}$ exist then the $P_{N}$ given by the equalities (23)-(25) are automatically a steady state ( $\mathrm{d} P_{N} / \mathrm{d} t=0$ ), since on substituting (23)-(25) into (22), the coefficients of $x_{\tau_{1}}$ cancel and the sums add up to zero. Thus, the problem is reduced to finding matrices $D, E$ and vectors $|V\rangle,\langle W|$ which satisfy the equalities (23)-(25) when we replace $P_{N}$ (or $f_{N}$ ) by their expressions (7). In this way we obtain the following conditions on $D$ and $E$ :

$$
\begin{aligned}
& \alpha\langle W| E=x_{1}\langle W|=-x_{0}\langle W| \\
& D E=-x_{0} D+x_{1} E \\
& \beta D|V\rangle=x_{1}|V\rangle=-x_{0}|V\rangle
\end{aligned}
$$

which are the same as (8)-(10) when

$$
x_{0}=-x_{1}=1
$$

The choice (29) is, in fact, not restrictive since (26), (27) imply that $x_{1}=-x_{0}$ and that the value of $x_{1}$ can be changed arbitrarily by multiplying $D$ and $E$ by a constant. 


\section{Size and forms of the matrices}

The question remains as to whether one can construct matrices $D$ and $E$ and vectors $|V\rangle$ and $\langle W|$ which satisfy (8)-(10). First of all one must know what size the matrices ought to be. We are going to show that they are either one-dimensional (when $\alpha+\beta=1$ ) or infinite-dimensional (when $\alpha+\beta \neq 1$ ). Let us distinguish two cases depending on whether $D$ and $E$ commute or not.

(i) If $D$ and $E$ commute, then (8)-(10) imply that

$$
\frac{1}{\alpha \beta}\langle W \mid V\rangle=\langle W|D E| V\rangle=\langle W|D+E| V\rangle=\left(\frac{1}{\alpha}+\frac{1}{\beta}\right)\langle W \mid V\rangle
$$

Therefore if $\langle W \mid V\rangle \neq 0$, one must have $\alpha+\beta=1$. So in that case, $D$ and $E$ can be chosen to be one-dimensional with $D=1 / \beta$ and $E=1 / \alpha$. This is a known case (Derrida et al 1992) where correlations are absent.

(ii) If $D$ and $E$ do not commute, one can show that the matrices $D$ and $E$ must be infinite as follows. Suppose that $D$ and $E$ were finite-dimensional. Then, since no non-zero vector $|v\rangle$ exists such that $E|v\rangle=|v\rangle$ (otherwise $D|v\rangle=D E|v\rangle=$ $(D+E)|v\rangle=D|v\rangle+|v\rangle$ which implies that $|v\rangle=0), E-1$ would be invertible . In that case one could calculate $D$ from relation (8) to find $D=E(E-1)^{-1}$ which would commute with $E$, in contradiction with the hypothesis. So when $\alpha+\beta \neq 1$, the only possibility left is to choose infinite-dimensional matrices.

There are several possible choices for the matrices $D, E$. One can use $|V\rangle, E|V\rangle, E^{2}|V\rangle \ldots$ as a basis for the infinite dimensional vector space, then $D$ and $E$ read

$$
D_{1}=\left(\begin{array}{ccccc}
1 / \beta & 1 / \beta & 1 / \beta & 1 / \beta & \cdots \\
0 & 1 & 1 & 1 & \\
0 & 0 & 1 & 1 & \\
0 & 0 & 0 & 1 & \\
\vdots & & & & \ddots
\end{array}\right) \quad E_{1}=\left(\begin{array}{ccccc}
0 & 0 & 0 & 0 & \cdots \\
1 & 0 & 0 & 0 & \\
0 & 1 & 0 & 0 & \\
0 & 0 & 1 & 0 & \\
\vdots & & & & \ddots
\end{array}\right) .
$$

For this choice of matrices, it is easy to check that

$$
\left\langle W_{1}\left|=\left(1,\left(\frac{1}{\alpha}\right),\left(\frac{1}{\alpha}\right)^{2} \cdots\right) \quad\right| V_{1}\right\rangle=\left(\begin{array}{c}
1 \\
0 \\
0 \\
\vdots
\end{array}\right)
$$

satisfy (8)-(10) and ensure that $\left\langle W_{1} \mid V_{1}\right\rangle=1$.

There are several other possible choices of matrices $D, E$ and vectors $\langle W|| V$, that satisfy (9)-(10). We can use this freedom to make the calculation of matrix elements more straightforward. For example, one can take

$$
D_{2}=\left(\begin{array}{ccccc}
1 & 1 & 0 & 0 & \cdots \\
0 & 1 & 1 & 0 & \\
0 & 0 & 1 & 1 & \\
0 & 0 & 0 & 1 & \\
\vdots & & & & \ddots
\end{array}\right) \quad E_{2}=\left(\begin{array}{ccccc}
1 & 0 & 0 & 0 & \cdots \\
1 & 1 & 0 & 0 & \\
0 & 1 & 1 & 0 & \\
0 & 0 & 1 & 1 & \\
\vdots & & & & \ddots
\end{array}\right)
$$




$$
\left\langle W_{2}\left|=\kappa\left(1,\left(\frac{1-\alpha}{\alpha}\right),\left(\frac{1-\alpha}{\alpha}\right)^{2} \cdots\right) \quad\right| V_{2}\right\rangle=\kappa\left(\begin{array}{c}
\left(\frac{1-\beta}{\beta}\right) \\
\left(\frac{1-\beta}{\beta}\right)^{2} \\
\vdots
\end{array}\right)
$$

where $\kappa=\sqrt{(\alpha+\beta-1) / \alpha \beta}$ to ensure that $\left\langle W_{2} \mid V_{2}\right\rangle=1$. In contrast to (31), (32) this choice makes the particle-hole symmetry of the problem apparent since the matrices $D_{2}, E_{2}$ have very similar forms and the boundary conditions $\alpha$ and $\beta$ only appear in the vectors $\left\langle W_{2}\right|$ and $\left|V_{2}\right\rangle$. For choice (33) of $D_{2}, E_{2}$ the elements of $C_{2}^{N}$ (where $C_{2}=D_{2}+E_{2}$ and $N$ denotes the $N$ th power of matrix $C_{2}$ ) are given by

$$
\left(C_{2}^{N}\right)_{n m}=\left(\begin{array}{c}
2 N \\
N+n-m
\end{array}\right)-\left(\begin{array}{c}
2 N \\
N+n+m
\end{array}\right) \text {. }
$$

Expression (35) can be obtained by noting that $\left(C_{2}^{N}\right)_{n m}$ is proportional to the probability that a random walker, who starts at site $2 m$ of a semi-infinite chain with absorbing boundary at the origin, is at site $2 n$ after $2 N$ steps of a random walk. A disadvantage of this choice (33), (34) is that, due to the form of $\left\langle W_{2}\right|$ and $\left|V_{2}\right\rangle$, one has to sum geometric series to obtain the correlation functions and these series diverge in some range of $\alpha, \beta$ (in fact $\alpha+\beta \leqslant 1$ ). However, at least for finite $N$, all expressions are rational functions of $\alpha, \beta$ so that one can obtain results for $\alpha+\beta \leqslant 1$ by analytic continuation from those for $\alpha+\beta>1$.

A third possible choice of $D, E,\langle W|| V$,$\rangle , that avoids these divergences, is$

$$
\begin{gathered}
D_{3}=\left(\begin{array}{ccccc}
1 / \beta & a & 0 & 0 & \cdots \\
0 & 1 & 1 & 0 & \\
0 & 0 & 1 & 1 & \\
0 & 0 & 0 & 1 & \ddots \\
\vdots & & & \ddots
\end{array}\right) \quad E_{3}=\left(\begin{array}{ccccc}
1 / \alpha & 0 & 0 & 0 & \cdots \\
a & 1 & 0 & 0 & \\
0 & 1 & 1 & 0 & \\
0 & 0 & 1 & 1 & \\
\vdots & & & \ddots & \ddots
\end{array}\right) \\
\\
\left\langle W_{3}|=(1,0,0, \ldots) \quad| V_{3}\right\rangle=\left(\begin{array}{c}
1 \\
0 \\
0 \\
\vdots
\end{array}\right)
\end{gathered}
$$

where

$$
a^{2}=\frac{\alpha+\beta-1}{\alpha \beta}
$$

The fact that $a^{2}$ may be negative is of no importance, because in the calculation of matrix elements we require later $a$ only enters through $a^{2}$. One should note that for $\alpha=\beta=1$, we have $a=1$ and (36), (37) coincides with our previous bidiagonal choice (33), (34). Also, one can see that for $\alpha+\beta=1$ we have $a=0$ so that the 1,1 elements of the matrices $D_{3}, E_{3}$ decouple from the other elements. This choice of matrices then becomes, for the purposes of our calculations, one-dimensional as is sufficient for this special case of $\alpha$ and $\beta$. 


\section{Exact results for finite systems}

In the previous section we presented some possible forms for the matrices and vectors $D, E,\langle W|| V$,$\rangle . However it is possible to obtain expressions for the densities and$ higher correlation functions directly from (8)-(11) without needing particular forms for the matrices. As we saw in (13) and (14) the calculation of any quantity of interest requires first expressions of $\left\langle W\left|C^{N}\right| V\right\rangle$ (where $C=D+E$ ) for arbitrary $N$ and for general values of $\alpha$ and $\beta$. There are several ways of calculating these matrix elements. One could (as shown in appendix B) diagonalize the matrix $C$ given by (31) or (33) or (36) and decompose the vectors $\langle W|| V$,$\rangle onto the eigenbasis of C$. Alternatively one could use generating function methods. Here we give an expression for $\left\langle W\left|C^{N}\right| V\right\rangle$, valid for $N \geqslant 1$, that can be obtained (see appendix $A$, section $\mathrm{A} 1$, equation (A12)) directly from the commutation rules (8)-(10):

$$
\left\langle W\left|C^{N}\right| V\right\rangle=\sum_{p=1}^{N} \frac{p(2 N-1-p) !}{N !(N-p) !} \frac{(1 / \beta)^{p+1}-(1 / \alpha)^{p+1}}{(1 / \beta)-(1 / \alpha)}
$$

where, as is our convention, the normalization is fixed to be $\langle W \mid V\rangle=1$. It is easy to check the first few cases:

$$
\begin{aligned}
\left\langle W\left|C^{N}\right| V\right\rangle= & \alpha^{-1}+\beta^{-1} \quad \text { for } N=1 \\
= & \alpha^{-2}+\alpha^{-1} \beta^{-1}+\beta^{-2}+\alpha^{-1}+\beta^{-1} \quad \text { for } N=2 \\
= & \alpha^{-3}+\alpha^{-2} \beta^{-1}+\alpha^{-1} \beta^{-2}+\beta^{-3} \\
& +2\left(\alpha^{-2}+\alpha^{-1} \beta^{-1}+\beta^{-2}+\alpha^{-1}+\beta^{-1}\right) \quad \text { for } N=3
\end{aligned}
$$

against direct calculation from $(8)-(10)$ or $(36)-(38)$.

To calculate the density profile and higher correlation functions it is convenient to use the following relation which is also a direct consequence of (8) and (11) (see appendix $A$, section $A 2$ ).

$$
D C^{n}=\sum_{p=0}^{n-1} \frac{2 p !}{p !(p+1) !} C^{n-p}+\sum_{p=2}^{n+1} \frac{(p-1)(2 n-p) !}{n !(n+1-p) !} D^{p}
$$

This relation is valid for all $n \geqslant 1$. It can easily be checked for $n=1,2,3$

$$
\begin{aligned}
& D C=C+D^{2} \quad D C^{2}=C^{2}+C+D^{2}+D^{3} \\
& D C^{3}=C^{3}+C^{2}+2 C+2 D^{2}+2 D^{3}+D^{4}
\end{aligned}
$$

by just applying the algebraic rules (11) and (8). By using the expression (13) for $\left\langle\tau_{i}\right\rangle_{N}$ and the fact that $D^{p}|V\rangle=\beta^{-p}|V\rangle$, one obtains for $i \leqslant N-1$

$$
\left\langle\tau_{i}\right\rangle_{N}=\sum_{p=0}^{n-1} \frac{2 p !}{p !(p+1) !} \frac{\left\langle W\left|C^{N-1-p}\right| V\right\rangle}{\left\langle W\left|C^{N}\right| V\right\rangle}+\frac{\left\langle W\left|C^{i-1}\right| V\right\rangle}{\left\langle W\left|C^{N}\right| V\right\rangle} \sum_{p=2}^{n+1} \frac{(p-1)(2 n-p) !}{n !(n+1-p) !} \beta^{-p}
$$


with $n=N-i$. The case $i=N$ has to be treated separately to give

$$
\left\langle\tau_{i}\right\rangle_{N}=\frac{1}{\beta} \frac{\left\langle W\left|C^{N-1}\right| V\right\rangle}{\left\langle W\left|C^{N}\right| V\right\rangle} .
$$

Thus (43), together with (39), yields exact expressions for the density profiles for arbitrary $N$ and general $\alpha$ and $\beta$.

Relation (41) can also be used to express the two-point functions $\left\langle\tau_{i} \tau_{j}\right\rangle_{N}$ for $1 \leqslant i<j \leqslant N-1$ in terms of one-point functions:

$$
\begin{aligned}
\left\langle\tau_{i} \tau_{j}\right\rangle_{N}=\sum_{p=0}^{n-1} & \frac{2 p !}{p !(p+1) !}\left\langle\tau_{i}\right\rangle_{n-p+j-1} \frac{\left\langle W\left|C^{n+j-1-p}\right| V\right\rangle}{\left\langle W\left|C^{N}\right| V\right\rangle} \\
& \quad+\left\langle\tau_{i}\right\rangle_{j-1} \frac{\left\langle W\left|C^{j-1}\right| V\right\rangle}{\left\langle W\left|C^{N}\right| V\right\rangle} \sum_{p=2}^{n+1} \frac{(p-1)(2 n-p) !}{n !(n+1-p) !} \beta^{-p}
\end{aligned}
$$

where $n=N-j$, and could similarly be used to obtain any higher order correlation functions.

In this section we have presented our main results and have shown that the matrix method easily yields exact expressions of all correlation functions for arbitrary $N, \alpha, \beta$. The following sections $6-9$ are concerned with finding various limits of these results as $N \rightarrow \infty$ which will, among other things, allow the phase diagram to be derived.

\section{The asymptotic forms of $\left\langle W\left|C^{N}\right| V\right\rangle$}

For large $N$, it is useful to have at our disposal the asymptotic forms of $\left\langle W\left|C^{N}\right| V\right\rangle$. These can be obtained by calculating the sum $R_{n}(x)$ defined by

$$
R_{n}(x)=\sum_{p=2}^{n+1} \frac{(p-1)(2 n-p) !}{n !(n+1-p) !} x^{p}
$$

relevant because (39) can be written as

$$
\left\langle W\left|C^{n}\right| V\right\rangle=\frac{R_{n}(1 / \beta)-R_{n}(1 / \alpha)}{(1 / \beta)-(1 / \alpha)} .
$$

For large $n$, the asymptotic form of $R_{n}(1 / \beta)$ can be obtained by looking at the values of $p$ which dominate the sum ( $p$ of order 1 for $\beta>\frac{1}{2}, p$ of order $n^{1 / 2}$ for $\beta=\frac{1}{2}$ and $p \simeq(1-2 \beta) /(1-\beta) n$ for $\left.\beta<\frac{1}{2}\right)$.

$$
\begin{aligned}
R_{n}\left(\frac{1}{\beta}\right)=\sum_{p=2}^{n+1} \frac{(p-1)(2 n-p) !}{n !(n+1-p) !} \frac{1}{\beta^{p}} & \simeq \frac{1}{\sqrt{\pi(2 \beta-1)^{2}}} \frac{4^{n}}{n^{3 / 2}} \quad \text { for } \beta>\frac{1}{2} \\
& \simeq \frac{2}{\sqrt{\pi}} \frac{4^{n}}{n^{1 / 2}} \quad \text { for } \beta=\frac{1}{2} \\
& \simeq(1-2 \beta)\left(\frac{1}{\beta(1-\beta)}\right)^{n+1} \quad \text { for } \beta<\frac{1}{2} .
\end{aligned}
$$


These expressions allow one to determine the following asymptotic forms of $\left\langle W\left|C^{N}\right| V\right\rangle$ for large $N$. Note that since (47) is symmetric in $\alpha$ and $\beta$, the cases where $\beta<\alpha$ can be obtained from those where $\alpha<\beta$ by interchanging $\alpha$ and $\beta$.

(a) For $\frac{1}{2}<\alpha<\beta$

$$
\left\langle W\left|C^{N}\right| V\right\rangle \simeq \frac{\alpha \beta}{\sqrt{\pi}(\beta-\alpha)}\left[\frac{1}{(2 \alpha-1)^{2}}-\frac{1}{(2 \beta-1)^{2}}\right] \frac{4^{N}}{N^{3 / 2}} .
$$

$\left(a^{\prime}\right)$ For $\frac{1}{2}<\alpha=\beta$

$$
\left\langle W\left|C^{N}\right| V\right\rangle \simeq \frac{\alpha^{2}}{\sqrt{\pi}(2 \alpha-1)^{3}} \frac{4^{N+1}}{N^{3 / 2}} .
$$

(b) For $\alpha=\frac{1}{2}<\beta$

$$
\left\langle W\left|C^{N}\right| V\right\rangle \simeq \frac{2 \beta}{\sqrt{\pi}(2 \beta-1)} \frac{4^{N}}{N^{1 / 2}} .
$$

$\left(b^{\prime}\right)$ For $\alpha=\beta=\frac{1}{2}$

$$
\left\langle W\left|C^{N}\right| V\right\rangle=4^{N} .
$$

(c) For $\alpha<\frac{1}{2}$ and $\alpha<\beta$

$$
\left\langle W\left|C^{N}\right| V\right\rangle \simeq \frac{\beta(1-2 \alpha)}{(\beta-\alpha)(1-\alpha)} \frac{1}{\alpha^{N}(1-\alpha)^{N}} .
$$

(d) For $\alpha=\beta<\frac{1}{2}$

$$
\left\langle W\left|C^{N}\right| V\right\rangle \simeq \frac{(1-2 \alpha)^{2}}{(1-\alpha)^{2}} \frac{N}{\alpha^{N}(1-\alpha)^{N}} .
$$

(The case $\left(b^{\prime}\right) \alpha=\beta=\frac{1}{2}$ is in fact trivial, as mentioned above, since $\alpha+\beta=1$ and $C=(1 / \alpha)+(1 / \beta)$ is a number. $)$

\section{The current and the phase diagram}

Recall that in section 2 we saw that, in the steady state, the current through the bond $i$ is simply $J=\left\langle\tau_{i}\left(1-\tau_{i+1}\right)\right\rangle$, because during a time interval $\mathrm{d} t$, the probability that a particle jumps from $i$ to $i+1$ is $\tau_{i}\left(1-\tau_{i+1}\right) \mathrm{d} t$. Therefore, $J$ is given by

$$
J=\frac{\left\langle W\left|C^{i-1} D E C^{N-i-1}\right| V\right\rangle}{\left\langle W\left|C^{N}\right| V\right\rangle}=\frac{\left\langle W\left|C^{N-1}\right| V\right\rangle}{\left\langle W^{N} C^{N} \mid V\right\rangle}
$$

where we have used the fact (11) that $D E=C$. We note that this expression is independent of $i$, as expected in the steady state.

From the asymptotic forms of $\left\langle W\left|C^{N}\right| V\right\rangle$, obtained in section 6 , it is easy to see that in the limit $N \rightarrow \infty$ 
(i) for $\alpha \geqslant \frac{1}{2}$ and $\beta \geqslant \frac{1}{2}$

$$
J=\frac{1}{4}
$$

(ii) for $\alpha<\frac{1}{2}$ and $\beta>\alpha$

$$
J=\alpha(1-\alpha)
$$

(iii) for $\beta<\frac{1}{2}$ and $\alpha>\beta$

$$
J=\beta(1-\beta) .
$$

Thus, the phase diagram consists of three phases (see figure 1): $\alpha>\frac{1}{2}, \beta>\frac{1}{2} ; \alpha<\frac{1}{2}$, $\beta>\alpha ; \beta<\frac{1}{2}, \alpha>\beta$. The same phase diagram was obtained by Andjel et al (1988) who considered an infinite system and then studied the long time evolution starting with the initial conditions of density $\alpha$ on the negative axis and density $1-\beta$ on the positive axis. It also turns out that the phase diagram is correctly given by mean-field approximations (Krug 1991, Derrida et al 1992). Equation (58) corresponds to the maximal-current phase whereas (59), (60) correspond, respectively, to the low- and high-density phases as will become obvious in the next section.

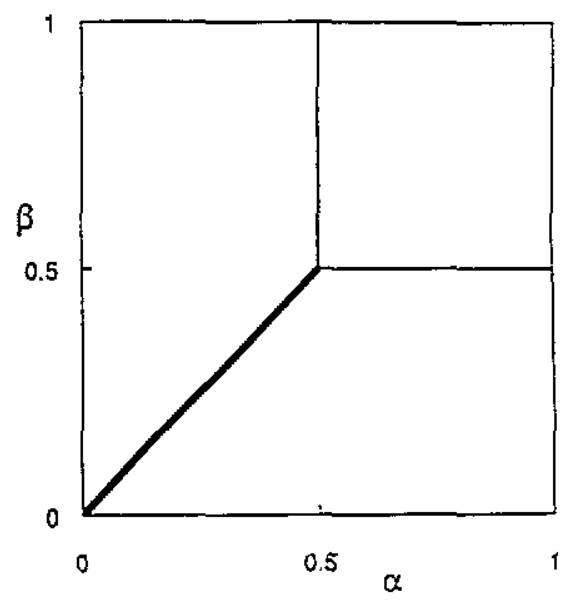

Figure 1. Phase diagram of the model. The region $\alpha>\frac{1}{2}$ and $\beta>\frac{1}{2}$ is the maximal current phase, whereas $\alpha<\frac{1}{2}$ and $\beta>\alpha$ is the low-density phase and $\beta<\frac{1}{2}$ and $\alpha>\beta$ is the high-density phase. The line $\alpha=\beta<\frac{1}{2}$ is a first-order transition line.

\section{The density $\left\langle\tau_{i}\right\rangle_{N}$ far from the boundaries}

From relation (43) and from the asymptotic forms (51)-(56) of $\left\langle W\left|C^{N}\right| V\right\rangle$, it is possible to obtain the asymptotic form of $\left\langle\tau_{i}\right\rangle_{N}$ in the limit $N \rightarrow \infty$, with $i=N x$ and $0<x<1$ (i.e. for $N$ large and $i$ in the bulk, far from both boundaries). 
In all cases (51)-(56), we have seen that $\left\langle W\left|C^{N}\right| V\right\rangle$ has the following asymptotic behaviour for large $N$ :

$$
\left\langle W\left|C^{N}\right| V\right\rangle=A N^{z} \lambda^{N}
$$

where $A, z$ and $\lambda$ depend on $\alpha$ and $\beta$ as in (51)-(56). This implies that for $N$ large and $i$ far from the boundaries

$$
\left\langle\tau_{i}\right\rangle_{N} \simeq \sum_{p=0}^{n-1} \frac{2 p !}{p !(p+1) !} \frac{1}{\lambda^{p+1}}+\frac{x^{z}}{\lambda^{n+1}} \sum_{p=2}^{n+1} \frac{(p-1)(2 n-p) !}{n !(n+1-p) !} \frac{1}{\beta^{p}}
$$

where $n=N-i$. For large $n$ (and $\lambda \geqslant 4$ which is always the case), the first term in (62) converges to $(1-\sqrt{1-4 / \lambda}) / 2$ and using the asymptotic forms (48)-(50) of the last sum in (62) one obtains that

(i) for $\alpha \geqslant \frac{1}{2}$ and $\beta \geqslant \frac{1}{2}$, the first term in (62) dominates and

$$
\left\langle\tau_{N x}\right\rangle_{N} \simeq \frac{1}{2}
$$

(ii) for $\alpha<\frac{1}{2}$ and $\alpha<\beta$, the first term in (62) dominates and

$$
\left\langle\tau_{N x}\right\rangle_{N} \simeq \alpha
$$

(iii) for $\beta<\frac{1}{2}$ and $\beta<\alpha$, both terms in (62) contribute and

$$
\left\langle\tau_{N x}\right\rangle_{N} \simeq 1-\beta
$$

(iv) for $\alpha=\beta<\frac{1}{2}$, both terms in (62) contribute and

$$
\left\langle\tau_{N x}\right\rangle_{N} \simeq \alpha+x(1-2 \alpha) .
$$

So everywhere in the phase diagram, the profile $\left\langle\tau_{i}\right\rangle_{N}$ is constant for $i$ far from the boundaries except along the first-order transition line $\alpha=\beta<\frac{1}{2}$ where the profile is linear (66). In the maximal current phase (63) the density is $\frac{1}{2}$. The region $\alpha<\frac{1}{2}$ and $\alpha<\beta$ corresponds to the low-density phase whereas the region $\beta<\frac{1}{2}$ and $\beta<\alpha$ corresponds to the high-density phase. There is a jump in the density when one crosses the first-order transition line $\alpha=\beta<\frac{1}{2}$.

The result that for $\alpha=\beta<\frac{1}{2}$ the profile is linear is due to the fact (Andjel et al 1988) that along this first-order line, there is a superposition of states where a shock between a left-hand region of density $\alpha$ and a right-hand region of density $1-\beta$ is present at an arbitrary position.

\section{The profile $\left\langle\tau_{i}\right\rangle_{N}$ near the boundaries}

Another case one can consider is the profile near a boundary when $N$ becomes large. First we note that there is an obvious particle-hole symmetry in the problem: since particles are injected at the left end with probability $\alpha$ and are removed at the right end with probability $\beta$, it is equivalent to saying that holes are injected at the right 
end with probability $\beta$ and they are removed at the left end with probability $\alpha$. Because of this particle-hole symmetry, one always has

$$
\left\langle\tau_{i}\right\rangle_{N}(\alpha, \beta)=1-\left\langle\tau_{N+1-i}\right\rangle_{N}(\beta, \alpha)
$$

which can be seen by using, for example, the representation $(33,34)$ and the fact that $D$ and $E$ are the transpositions of each other. So one can do all the calculations for the right boundary, then by using this symmetry deduce what happens at the left boundary. Starting from (43), and choosing $n=N-i$ finite as $N$ goes to infinity, one finds for $n \geqslant 1$

$$
\left\langle\tau_{N-n}\right\rangle_{N}=\sum_{p=0}^{n-1} \frac{2 p !}{p !(p+1) !} \frac{1}{\lambda^{p+1}}+\frac{1}{\lambda^{n+1}} \sum_{p=2}^{n+1} \frac{(p-1)(2 n-p) !}{n !(n+1-p) !} \frac{1}{\beta^{p}}
$$

where from (51)-(56)

$$
\begin{array}{ll}
\lambda=4 & \text { for } \alpha \geqslant \frac{1}{2} \text { and } \beta \geqslant \frac{1}{2} \\
\lambda=[\alpha(1-\alpha)]^{-1} & \text { for } \alpha \leqslant \frac{1}{2} \text { and } \alpha \leqslant \beta \\
\lambda=[\beta(1-\beta)]^{-1} & \text { for } \beta \leqslant \frac{1}{2} \text { and } \alpha \geqslant \beta
\end{array}
$$

The special case $n=0$ is easy to treat directly from (13)

$$
\left\langle\tau_{N}\right\rangle_{N}=\frac{\left\langle W\left|C^{N-1} D\right| V\right\rangle}{\left\langle W\left|C^{N}\right| V\right\rangle}=\frac{1}{\lambda \beta} .
$$

Then using the symmetry property (67) and the fact that $\lambda$ is a symmetric function of $\alpha$ and $\beta$, one finds for the left boundary in the limit of $N \rightarrow \infty$ for $n \geqslant 2$

$$
\left\langle\tau_{n}\right\rangle_{N}=1-\sum_{p=0}^{n-2} \frac{2 p !}{p !(p+1) !} \frac{1}{\lambda^{p+1}}-\frac{1}{\lambda^{n}} \sum_{p=2}^{n} \frac{(p-1)(2 n-2-p) !}{(n-1) !(n-p) !} \frac{1}{\alpha^{p}}
$$

and for $n=1$

$$
\left\langle\tau_{1}\right\rangle_{N}=1-\frac{1}{\alpha \lambda} .
$$

To obtain the large $n$ behaviour of these expressions (keeping $n \ll N$ ), one needs in addition to the asymptotic forms (48)-(50) of $R_{n}(x)$ defined by (46), the asymptotic forms of the sum $S_{n}(x)$ defined by

$$
S_{n}(x)=\sum_{q=0}^{n-1} \frac{(2 q) !}{(q+1) ! q !} x^{q}=\frac{1-\sqrt{1-4 x}}{2 x}-\sum_{q=n}^{\infty} \frac{(2 q) !}{(q+1) ! q !} x^{q} \quad \text { for } x \leqslant \frac{1}{4} .
$$

For large $n$, one can show that for $x<\frac{1}{4}$

$$
S_{n}(x)=\frac{1-\sqrt{1-4 x}}{2 x}-\frac{(4 x)^{n}}{\sqrt{\pi} n^{3 / 2}} \frac{1}{1-4 x}+\cdots
$$


for $x=\frac{1}{4}$

$$
S_{n}(x)=2-\frac{2}{\sqrt{\pi} n^{1 / 2}}+\frac{1}{4 \sqrt{\pi} n^{3 / 2}}+\cdots .
$$

Let us now describe the profile at the right boundary when $N \rightarrow \infty$. Using (46) and (73), one can rewrite (68) as

$$
\left\langle\tau_{N-n}\right\rangle_{N}=\frac{1}{\lambda} S_{n}\left(\frac{1}{\lambda}\right)+\frac{1}{\lambda^{n+1}} R_{n}\left(\frac{1}{\beta}\right)
$$

and from the knowledge (70) of $\lambda$, and of the asymptotic forms (48)-(50), (74), (75) of $R_{n}$ and $S_{n}$, one obtains for the sites close to the right boundary as follows.

In the maximal-current phase, i.e. for $\alpha \geqslant \frac{1}{2}$ and $\beta \geqslant \frac{1}{2}$,

$$
\begin{aligned}
& \left\langle\tau_{N}\right\rangle_{N}=\frac{1}{4 \beta} \\
& \left\langle\tau_{N-1}\right\rangle_{N}=\frac{1}{4}+\frac{1}{16 \beta^{2}} \\
& \left\langle\tau_{N-2}\right\rangle_{N}=\frac{5}{16}+\frac{1}{64 \beta^{2}}+\frac{1}{64 \beta^{3}}
\end{aligned}
$$

$\left\langle\tau_{N-n}\right\rangle_{N} \simeq \frac{1}{2}-\frac{1}{2 \sqrt{\pi} n^{1 / 2}}+\frac{(2 \beta-1)^{2}+4}{16 \sqrt{\pi}(2 \beta-1)^{2} n^{3 / 2}}+\cdots \quad$ for large $n$ and $\beta>\frac{1}{2}$ $\left\langle\tau_{N-n}\right\rangle_{N}=\frac{1}{2} \quad$ for large $n$ and $\beta=\frac{1}{2}$.

In the low-density phase, i.e. for $\alpha<\frac{1}{2}$ and $\alpha \leqslant \beta$,

$$
\begin{aligned}
& \left\langle\tau_{N}\right\rangle_{N}=\alpha(1-\alpha) / \beta \\
& \left\langle\tau_{N-1}\right\rangle_{N}=\alpha(1-\alpha)\left(1+\alpha(1-\alpha) / \beta^{2}\right) \\
& \left\langle\tau_{N-2}\right\rangle_{N}=\alpha(1-\alpha)\left(1+\alpha(1-\alpha)+\alpha^{2}(1-\alpha)^{2}\left(\frac{1}{\beta^{2}}+\frac{1}{\beta^{3}}\right)\right) \\
& \quad \vdots \\
& \left\langle\tau_{N-n}\right\rangle_{N} \simeq \alpha+4^{n}(\alpha(1-\alpha))^{n+1} \frac{1}{\sqrt{\pi} n^{3 / 2}}\left(\frac{1}{(1-2 \beta)^{2}}-\frac{1}{(1-2 \alpha)^{2}}\right)
\end{aligned}
$$

for large $n$ and $\beta>\frac{1}{2}$

$\left\langle\tau_{N-n}\right\rangle_{N} \simeq \alpha+4^{n}(\alpha(1-\alpha))^{n+1} \frac{2}{\sqrt{\pi} n^{1 / 2}} \quad$ for large $n$ and $\beta=\frac{1}{2}$

$\left\langle\tau_{N-n}\right\rangle_{N} \simeq \alpha+\left(\frac{\alpha(1-\alpha)}{\beta(1-\beta)}\right)^{n+1}(1-2 \beta) \quad$ for large $n$ and $\alpha \leqslant \beta<\frac{1}{2}$.

In the high-density phase, i.e. for $\beta<\frac{1}{2}$ and $\beta \leqslant \alpha$,

$$
\left\langle\tau_{N}\right\rangle_{N}=\left\langle\tau_{N-1}\right\rangle_{N}=\left\langle\tau_{N-2}\right\rangle_{N}=\left\langle\tau_{N-n}\right\rangle_{N} \cdots=1-\beta
$$


Similar expressions could easily be obtained for the left boundary, using the symmetry (67). We see that in one phase (77) we have power-law decays of the profiles near the boundary, whereas in the other two phases (78) and (79) the profile decays exponentially at one boundary and is constant near the other. Thus, in the latter two phases one of the boundary conditions dominates and the profile depends on this boundary condition at the opposite end of the system, even when the system is of infinite length. Finally, it is worth noting that for $\alpha+\beta=1$ one recovers the fact that $\left\langle\tau_{i}\right\rangle_{N}=\alpha$, independent of $i$.

\section{The case $\alpha=\beta=1$}

In this section we show that the matrix approach allows us to recover very easily some known results for the case $\alpha=\beta=1$ (Derrida et al 1992). In particular we shall derive a relation between correlation functions of different order that was conjectured in Derrida and Evans (1993).

The simplest way of obtaining $\left\langle W\left|C^{N}\right| V\right\rangle$ is to use matrices and vectors (33), (34). From (35) we have

$$
\left\langle W\left|C^{N}\right| V\right\rangle=\left(C_{2}^{N}\right)_{11}=\frac{(2 N) !}{N ! N !}-\frac{(2 N) !}{(N+2) !(N-2) !}=A(N+1)
$$

where $A(p)$ is defined by

$$
A(p)=\frac{(2 p) !}{(p+1) ! p !}
$$

Now using (33) for the matrices $D$ and $E$ and

$$
\langle W|=(1,0,0, \ldots) \quad| V\rangle=\left(\begin{array}{l}
1 \\
0 \\
0 \\
\vdots
\end{array}\right)
$$

it is easy to verify that

$$
D E-E D=D C-C D=|V\rangle\langle W| .
$$

Therefore from (13), one can see that

$$
\left\langle\tau_{i}\right\rangle_{N}-\left\langle\tau_{i+1}\right\rangle_{N}=\frac{\left\langle W\left|C^{i-1}(D C-C D) C^{N-i-1}\right| V\right\rangle}{\left\langle W\left|C^{N}\right| V\right\rangle}=\frac{A(i) A(N-i)}{A(N+1)}
$$

and using the fact that

$$
\left\langle\tau_{N}\right\rangle_{N}=\frac{\left\langle W\left|C^{N-1} D\right| V\right\rangle}{\left\langle W\left|C^{N}\right| V\right\rangle}=\frac{A(N)}{A(N+1)}
$$

one finds

$$
\left\langle\tau_{i}\right\rangle_{N}=\frac{1}{A(N+1)} \sum_{p=0}^{N-i} A(p) A(N-p)
$$


This is one of the expressions of the one-point correlation function derived in Derrida et al (1992).

Similarly if one considers a higher-order correlation function, one can show that

$$
\begin{aligned}
& \left\langle\tau_{k_{1}} \tau_{k_{2}} \ldots \tau_{k_{n-1}} \tau_{k_{n}}\right\rangle_{N}-\left\langle\tau_{k_{1}} \tau_{k_{2}} \ldots \tau_{k_{n-1}} \tau_{k_{n}+1}\right\rangle_{N} \\
& =\frac{A\left(N-k_{n}\right) A\left(k_{n}\right)}{A(N+1)}\left\langle\tau_{k_{1}} \tau_{k_{2}} \ldots \tau_{k_{n-1}}\right\rangle_{N-k_{n}-1}
\end{aligned}
$$

which leads to

$$
\left\langle\tau_{k_{1}} \tau_{k_{2}} \ldots \tau_{k_{n}}\right\rangle_{N}=\frac{1}{A(N+1)} \sum_{p=0}^{N-k_{n}} A(p) A(N-p)\left\langle\tau_{k_{1}} \tau_{k_{2}} \ldots \tau_{k_{n-1}}\right\rangle_{N-p-1} \text {. }
$$

This relation was established for pair correlations and conjectured for higher-order correlations in Derrida and Evans (1993) (although the notation there was slightly different).

\section{Generalizations and conclusion}

In this paper we have shown that the steady state of the asymmetric exclusion model can be described in a very simple way in terms of the non-commuting matrices $D$ and $E$ which satisfy (7)-(10). Using this technique, the calculation of the current, the densities, the profiles and the correlation functions is much easier than with the previously used approaches to the same problem. It is known that in the case of periodic boundaries (Dhar 1987, Gwa and Spohn 1992) or of parallel updating (Schütz 1992), the asymmetric exclusion model can be solved by means of the Bethe ansatz. Of course, it would be very instructive to better understand the link between the traditional Bethe ansatz approach and the matrix formulation we have used here.

Our matrix approach has allowed us to calculate new quantities and to rederive known results for the fully asymmetric model with open boundaries. What makes it, in our opinion, of further interest is that it can be extended to more general situations: the problem of partially asymmetric exclusion, and the case of a mixture of two kinds of particles. Let us conclude by discussing briefly how the essentials of our approach may be applied to these two of the possible generalizations.

\subsection{The partially asymmetric exclusion process}

The partially asymmetric exclusion process is a generalization of the problem to the case where particles have a probability of hopping to the left as well as of hopping to the right: during every infinitesimal time interval $\mathrm{d} t$, each particle on sites $2 \leqslant i \leqslant N-1$ in the system has a probability $p \mathrm{~d} t$ of jumping to the neighbouring site on its right (if this site is empty), and a probability $q \mathrm{~d} t$ of of jumping to the neighbouring site on its left (if this site is empty). The boundary conditions are defined as follows. A particle is added at site $i=1$ with probability $\alpha \mathrm{d} t$ if site 1 is empty; a particle at site one has a probability $\gamma \mathrm{d} t$ of being removed from the system and has a probability $p \mathrm{~d} t$ of jumping to the right if site two is empty; a particle at site $N$ is removed with probability $\beta \mathrm{d} t$ and has a probability $q \mathrm{~d} t$ of jumping to the 
left if site $N-1$ is empty; particles are added at site $N$ with probability $\delta \mathrm{d} t$ if site $N$ is empty.

For this system, the weights in the steady state $f_{N}\left(\tau_{1}, \tau_{2}, \ldots, \tau_{N}\right)$ can also be obtained through the expression

$$
f_{N}\left(\tau_{1}, \tau_{2}, \ldots, \tau_{N}\right)=\left\langle W\left|\prod_{i=1}^{N}\left(\tau_{i} D+\left(1-\tau_{i}\right) E\right\rangle\right| V\right\rangle
$$

where $D, E,|V\rangle$ and $\langle W|$ now satisfy

$$
\begin{aligned}
& p D E-q E D=D+E \\
& (\beta D-\delta E)|V\rangle=|V\rangle \\
& \langle W|(\alpha E-\gamma D)=\langle W| .
\end{aligned}
$$

Because of the left-right symmetry of the problem, we need only consider the case $p \geqslant q$. If we take $p=1, q=0, \gamma=0, \delta=0$ the model reduces to the fully asymmetric exclusion process we considered in the previous sections and (90)-(92) recover $(8)-(10)$.

The proof that (89)-(92) yield the steady state of the weights is analogous to the proof presented in section 3 , the only difference being that the transition matrices are modified to

$$
h_{1}=\left(\begin{array}{cc}
-\alpha & \gamma \\
\alpha & -\gamma
\end{array}\right) \quad h_{N}=\left(\begin{array}{cc}
-\delta & \beta \\
\delta & -\beta
\end{array}\right) \quad h=\left(\begin{array}{cccc}
0 & 0 & 0 & 0 \\
0 & -q & p & 0 \\
0 & q & -p & 0 \\
0 & 0 & 0 & 0
\end{array}\right) \text {. }
$$

We note that the elements of each column of these matrices add up to zero and this again creates the cancellation of terms required in the proof.

One can check that one-dimensional matrices suffice when

$$
(p-q)(\alpha+\delta)(\beta+\gamma)=(\alpha+\delta+\beta+\gamma)(\alpha \beta-\gamma \delta)
$$

otherwise infinite-dimensional matrices are required.

For general $\alpha, \beta, \gamma, \delta$, we did not find convenient closed expressions for matrices and vectors $D, E,\langle W|| V$,$\rangle that satisfy (90)-(92). However, we checked that the$ conditions on the stationary weights implied by (90)-(92) hold for system sizes $N=1,2,3$.

A situation where we did succeed in finding closed forms for the matrices and vectors was $\gamma=\delta=0$, i.e. when particles cannot enter at the right-hand boundary and cannot leave at the left-hand boundary. In this case one possible choice of matrices, convenient for $p \geqslant q$, is

$$
D=\frac{1}{p-q}\left(\begin{array}{ccccc}
1-d & a_{1} & 0 & 0 & \cdots \\
0 & 1-d(q / p) & a_{2} & 0 & \\
0 & 0 & 1-d(q / p)^{2} & a_{3} & \\
0 & 0 & 0 & 1-d(q / p)^{3} & \\
\vdots & & & & \ddots
\end{array}\right)
$$




$$
\begin{gathered}
E=\frac{1}{p-q}\left(\begin{array}{ccccc}
1-e & 0 & 0 & 0 & \cdots \\
a_{1} & 1-e(q / p) & 0 & 0 & \\
0 & a_{2} & 1-e(q / p)^{2} & 0 & \\
0 & 0 & a_{3} & 1-e(q / p)^{3} & \\
\vdots & & & \ddots .
\end{array}\right) \\
\langle W|=(1,0,0 \cdots) \\
|V\rangle=\left(\begin{array}{c}
1 \\
0 \\
0 \\
\vdots
\end{array}\right)
\end{gathered}
$$

where

$$
\begin{aligned}
& d=1-\frac{p-q}{\beta} \quad e=1-\frac{p-q}{\alpha} \\
& \left(a_{i}\right)^{2}=\left[1-\left(\frac{q}{p}\right)^{i}\right]\left[1-d e\left(\frac{q}{p}\right)^{i-1}\right] .
\end{aligned}
$$

We note that for $p \rightarrow q$ the limiting forms of matrices (95), (96) are well defined, and for $p=1, q=0$ the matrices and vectors reduce to the third choice (36), (37) that we presented for fully asymmetric exclusion.

\subsection{The case of second class particles}

There is a simple extension of the fully asymmetric exclusion model, the problem of a mixture of first- and second-class particles, which has been used recently to describe shocks (Boldrighini et al 1989, Ferrari et al 1991, Ferrari 1992). One version of the model consists of considering $K_{1}$ first-class particles, $K_{2}$ second-class particles on a ring of $N$ sites. Then during each time step d $t$, each first-class particle has a probability $\mathrm{d} t$ of jumping to its right provided that its nearest neighbour on the right is empty or occupied by a second-class particle. In the latter case, the second class particle jumps simultaneously to the left (in other words, the first- and the secondclass particle are exchanged). Also, during each time interval d $t$, each second-class particle has a probability $\mathrm{d} t$ of jumping to its right if its right neighbour is empty. So the second-class particles look like holes, as seen from first-class particles and they look like particles as seen from the holes.

It turns out that one can use the matrix formulation in this case too. The weight of a configuration is then given by

$$
\operatorname{tr}\left(X_{1} X_{2} \cdots X_{N}\right)
$$

where the matrix $X_{i}=D$ if site $i$ is occupied by a first-class particle, $X_{i}=E$ if site $i$ is empty and $X_{i}=A$ when site $i$ is occupied by a second-class particle. It can be shown (Derrida, Janovsky, Lebowitz and Speer 1993) that a sufficient condition for the matrices $D, E, A$ to give the right steady state is that

$$
D E=D+E \quad D A=A \quad A E=A \text {. }
$$

Using this matrix formulation, it is then possible to derive exact expressions for the profiles of shocks (Derrida, Janovsky, Lebowitz and Speer 1993). 


\section{Acknowledgments}

We are grateful to T Liggett, D Mukamel and E Saada for useful discussions. Special thanks are due to $\mathrm{C}$ Kipnis for his interest and help in guiding us through the mathematical literature.

\section{Appendix A. Proofs of formulae (39), (41)}

In this appendix we shall derive some useful formulae (39), (41) which are required in the main body of the paper. These formulae are direct consequences of the algebra (8) and (11)

$$
D E=D+E
$$

and

$$
C=D+E \text {. }
$$

Before deriving the aforementioned formulae, we state two elementary identities:

(i) for $0 \leqslant p \leqslant n+1$

$$
\frac{p(2 n+1-p) !}{(n+1) !(n+1-p) !}-\frac{(p+1)(2 n-p) !}{(n+1) !(n-p) !}=\frac{(p-1)(2 n-p) !}{n !(n+1-p) !}
$$

(where for $p=n+1$ we take the convention that $(n-p) !=\infty$ );

(ii) for $n \geqslant 0$

$$
D^{n} C=C+\sum_{q=2}^{n+1} D^{q}=\frac{D^{n+2}-D^{2}}{D-1}+C=\frac{D^{n+2}-D}{D-1}+E .
$$

Here, and in the following, $\left(D^{n+1}-D\right)(D-1)^{-1}$ is a shorthand notation for $D^{n}+D^{n-1}+\cdots+D$ so that the question of whether $(D-1)$ is invertible or not is unimportant. The first of these identities (A3) may be checked directly. Relation (A4) follows immediately from (A1) and (A2) when $n=0$ or 1 since $D C=D(D+E)=D^{2}+D+E=D^{2}+C$. It is then straightforward to verify (A4) by recursion on $n$.

\section{A1. Proof of relation (39)}

To prove this relation for $n \geqslant 1$, we shall first prove that

$$
C^{n}=\sum_{p=0}^{n} \frac{p(2 n-1-p) !}{n !(n-p) !} \sum_{q=0}^{p} E^{q} D^{p-q}
$$

which we check for $n=1$ and then prove by recursion on $n$. For $n=1$ (A5) reads $C=D+E$, which is known from (A2). If we assume that (A5) is true for $n$, we may calculate $C^{n+1}$ by right multiplication with $D+E$ to find

$$
C^{n+1}=\sum_{p=0}^{n} \frac{p(2 n-1-p) !}{n !(n-p) !} \sum_{q=0}^{p}\left[E^{q+1}+E^{q}\left(\frac{D^{p-q+2}-D}{D-1}\right)\right]
$$


where we have used (A4). We now rewrite (A6), by inserting (A3), as

$$
\begin{aligned}
C^{n+1}=\sum_{p=0}^{n} & \frac{(p+1)(2 n-p) !}{(n+1) !(n-p) !} \sum_{q=0}^{p}\left[E^{q+1}+E^{q}\left(\frac{D^{p-q+2}-D}{D-1}\right)\right] \\
& \quad-\sum_{p=0}^{n} \frac{(p+2)(2 n-p-1) !}{(n+1) !(n-p-1) !} \sum_{q=0}^{p}\left[E^{q+1}+E^{q}\left(\frac{D^{p-q+2}-D}{D-1}\right)\right] \\
= & \frac{(2 n) !}{(n+1) ! n !}(E+D)+\sum_{p=2}^{n+1} \frac{p(2 n+1-p) !}{(n+1) !(n-p+1) !} \\
& \times\left\{\sum_{q=0}^{p-1}\left[E^{q+1}+E^{q}\left(\frac{D^{p-q+1}-D}{D-1}\right)\right]\right. \\
& \left.\quad-\sum_{q=0}^{p-2}\left[E^{q+1}+E^{q}\left(\frac{D^{p-q}-D}{D-1}\right)\right]\right\}
\end{aligned}
$$

where, in going from (A7) to (A8), we have written separately the first term of the first sum over $p$ in (A7), then relabelled the first sum by $p \rightarrow p-1$ and the second sum by $p \rightarrow p-2$ to allow the two sums to be combined. From (A8) we easily obtain

$$
\begin{aligned}
C^{n+1}= & \frac{(2 n) !}{(n+1) ! n !}(E+D) \\
& \quad+\sum_{p=2}^{n+1} \frac{p(2 n+1-p) !}{(n+1) !(n+1-p) !}\left[E^{p}+\sum_{q=0}^{p-1} E^{q} D^{p-q}\right] \\
= & \sum_{p=0}^{n+1} \frac{p(2 n+1-p) !}{(n+1) !(n+1-p) !} \sum_{q=0}^{p} E^{q} D^{p-q} .
\end{aligned}
$$

This is in agreement with (A5), which is hence proven by recursion.

To obtain (39), we calculate the matrix elements $\left\langle W\left|C^{n}\right| V\right\rangle$, for $n \geqslant 1$ simply by using (A5) and the algebra $(9,10)$

$$
\left\langle W\left|C^{n}\right| V\right\rangle=\sum_{p=0}^{n} \frac{p(2 n-1-p) !}{n !(n-p) !} \sum_{q=0}^{p} \frac{1}{\alpha^{q}} \frac{1}{\beta^{p-q}}\langle W \mid V\rangle .
$$

The sum over $q$ may be performed as a geometric series and, as the normalization is $\langle W \mid V\rangle=1$, we obtain

$$
\left\langle W\left|C^{n}\right| V\right\rangle=\sum_{p=0}^{n} \frac{p(2 n-1-p) !}{n !(n-p) !} \frac{(1 / \beta)^{p+1}-(1 / \alpha)^{p+1}}{(1 / \beta)-(1 / \alpha)} \quad \text { for } n \geqslant 1 .
$$

We note again that (A12) has been derived directly from the algebra (8)-(10), without reference to any particular form of matrices. 


\section{A2. Proof of relation (41)}

By an approach similar to the previous derivation we are going to prove (41)

$$
D C^{n}=\sum_{p=0}^{n-1} \frac{2 p !}{p !(p+1) !} C^{n-p}+\sum_{p=2}^{n+1} \frac{(p-1)(2 n-p) !}{n !(n+1-p) !} D^{p} .
$$

For $n=1$ (A13) reads $D C=D^{2}+C$ which agrees with (A4). Assuming that (A13) is true for $n$ we check that it remains true for $n+1$ by right multiplying with $C$ to give

$$
\begin{aligned}
D C^{n+1}= & \sum_{p=0}^{n-1} \frac{2 p !}{p !(p+1) !} C^{n-p+1}+\sum_{p=2}^{n+1} \frac{(p-1)(2 n-p) !}{n !(n+1-p) !} \sum_{q=2}^{p+1} D^{q} \\
& +\sum_{p=2}^{n+1} \frac{(p-1)(2 n-p) !}{n !(n+1-p) !} C \\
= & \sum_{p=0}^{n-1} \frac{2 p !}{p !(p+1) !} C^{n-p+1}+\sum_{q=2}^{n+2} D^{g} \sum_{p=q-1}^{n+1} \frac{(p-1)(2 n-p) !}{n !(n+1-p) !} \\
& +C \sum_{p=2}^{n+1} \frac{(p-1)(2 n-p) !}{n !(n+1-p) !}
\end{aligned}
$$

where we have used the first equality of (A4) to obtain (A14) and we have reordered the sums in the second term of (A14) in going to (A15). Now, by summing (A3) it is straightforward to obtain the identity

$$
\sum_{p=q-1}^{n+1} \frac{(p-1)(2 n-p) !}{n !(n+1-p) !}=\frac{(q-1)(2 n+2-q) !}{(n+1) !(n+2-q) !},
$$

We use (A16) twice in (A15) to give

$$
D C^{n+1}=\sum_{p=0}^{n} \frac{2 p !}{p !(p+1) !} C^{n+1-p}+\sum_{p=2}^{n+2} \frac{(p-1)(2 n+2-p) !}{(n+1) !(n+2-p) !} D^{p}
$$

which is in agreement with (A13). Equation (A13) is hence proven by recursion.

Appendix B. Computation from a particular matrix representation; the example of the normalization $\left\langle W\left|C^{N}\right| V\right\rangle$

In the main part of this paper, expressions for the different steady-state characteristics, such as the average site occupation or mean current, have been derived directly from the matrix commutation rules (8)-(10), without using a form of the matrices. An alternative way to proceed is to choose a convenient representation of these matrices and to use it as fully as possible. We illustrate this second approach here, with the 
computation of the matrix element $\left\langle W\left|C^{N}\right| V\right\rangle$. We choose the representation (33), (34)

$$
C=\left(\begin{array}{ccccc}
2 & 1 & 0 & 0 & \ldots \\
1 & 2 & 1 & 0 & \\
0 & 1 & 2 & 1 & \\
0 & 0 & 1 & 2 & \\
\vdots & & & & \ddots
\end{array}\right)
$$

with

$$
\left\langle W\left|=\kappa\left(1, a, a^{2}, \cdots\right) \quad\right| V\right\rangle=\kappa\left(\begin{array}{c}
1 \\
b \\
b^{2} \\
\vdots
\end{array}\right)
$$

and $a=(1-\alpha) / \alpha, b=(1-\beta) / \beta, \kappa^{2}=(\alpha+\beta-1) / \alpha \beta$. A simple way to compute $\left\langle W\left|C^{N}\right| V\right\rangle$ is to represent $|V\rangle$ as a linear combination of eigenvectors of $C$ in order to evaluate easily $C^{N}|V\rangle$. It can be checked that $C|\theta\rangle=2(1+\cos \theta)|\theta\rangle$ where $|\theta\rangle$ is given by

$$
|\theta\rangle=\kappa\left(\begin{array}{c}
\sin \theta \\
\sin 2 \theta \\
\sin 3 \theta \\
\vdots
\end{array}\right)
$$

For $b<1$ (i.e. $\beta>\frac{1}{2}$ ), $|V\rangle$ can be written as a linear combination of these eigenvectors.

$$
|V\rangle=\kappa \int_{-\pi}^{+\pi} \frac{\mathrm{d} \theta}{\pi} L_{b}(\theta)|\theta\rangle
$$

with

$$
L_{b}(\theta)=\frac{\sin \theta}{1-2 b \cos \theta+b^{2}} .
$$

This readily follows from the identity, which can be derived by residues or looked up in Gradshstein and Ryzhyk (1980, formula (3.613-3))

$$
b^{k-1}=\int_{-\pi}^{+\pi} \frac{\mathrm{d} \theta}{\pi} \frac{\sin k \theta \sin \theta}{1-2 b \cos \theta+b^{2}} .
$$

Using the fact that $|\theta\rangle$ is an eigenvector of $C$ with eigenvalue $2(1+\cos \theta)$, it follows from (B4) that $C^{N}|V\rangle$ is

$$
C^{N}|V\rangle=\kappa \int_{-\pi}^{+\pi} \frac{\mathrm{d} \theta}{\pi}[2(1+\cos \theta)]^{N} L_{b}(\theta)|\theta\rangle .
$$


In order to complete the calculation it remains to evaluate $\langle W \mid \theta\rangle$. For $a<1$ (i.e. $\alpha>\frac{1}{2}$ ) this is

$$
\langle W \mid \theta\rangle=\sum_{k=1}^{\infty} a^{k-1} \sin k \theta=L_{a}(\theta) .
$$

The last equality is obtained by writing the sinus function as a sum of two exponentials and summing the resulting convergent series (using $a<1$ ). Finally, one obtains for $\alpha>\frac{1}{2}, \beta>\frac{1}{2}$

$$
\begin{aligned}
\left\langle W\left|C^{N}\right| V\right\rangle & =\kappa^{2} \int_{-\pi}^{+\pi} \frac{\mathrm{d} \theta}{\pi} L_{a}(\theta) L_{b}(\theta)[2(1+\cos \theta)]^{N} \\
& =\frac{\alpha+\beta-1}{\alpha \beta} \int_{-\pi}^{+\pi} \frac{\mathrm{d} \theta}{\pi} \frac{\sin ^{2} \theta[2(1+\cos \theta)]^{N}}{\left(1-2 a \cos \theta+a^{2}\right)\left(1-2 b \cos \theta+b^{2}\right)}
\end{aligned}
$$

As stated in the main text, results for other values of $\alpha$ and $\beta$ can be obtained by analytic continuation of (B10). This is conveniently done by writing (B10) as an integral around the unit circle in the complex plane and following the deformation of the integration contour as poles move in and out of the unit circle. The integral representation (B10) combined with the saddle-point method is then an alternative way to derive the large- $N$ asymptotic expressions (51)-(56) of the main text.

\section{References}

Andjel E D, Bramson M and Liggett T M 1988 Shocks in the asymmetric simple exciusion process Prob. Theory Rel Fields 78 231-47

Baxter R J 1982 Exactly Solved Models in Statistical Mechanics (New York: Academic)

Boldrighini C, Cosimi G, Frigio S and Grasso Nuñes M 1989 Computer simulation of shock waves in the completely asymmetric simple exclusion process J. Stat Phys. 55611

Bramson M 1988 Front propagation in certain one-dimensional exclusion models $J$. Stat. Phys. 51863

DeMasi A, Kipnis C, Presutti E and Saada E 1988 Microscopic structure at the shock in the simple asymmetric exclusion process Stochastics 27 151-65

Derrida B, Domany E and Mukamel D 1992 An exact solution of a one dimensional asymmetric exclusion model with open boundaries J. Stat. Phys. $69667-87$

Derrida B and Evans M R 1993 Exact correlation functions in an asymmetric exclusion model with open boundaries $J$. Physique I 3 311-22

Derrida B, Evans M R, Hakim V and Pasquier V 1993 A matrix method of solving an asymmetric exclusion model with open boundaries Cellular Automata and Cooperative Systems (Proc. Les Houches Workshop, June 1992) ed N Boccara, E Goles, S Martinez and P Picco to appear

Derrida B, Janowsky S A, Lebowitz J L and Speer E R 1993 in preparation

Dhar D 1987 An exactly solved model for interfacial growth Phase Transitions 951

Faddeev L D 1980 Quantum completely integrable models in field theory Sov. Sci. Rev C 1107

Fannes M, Nachtergaele B and Werner R F 1992 Finitely correlated states on quantum spin chains Commun. Math. Phys. 144443

Ferrari P A 1986 The simple exclusion process as seen from a tagged particle Ann Prob. 14 1277-90

— 1992 Shock fluctuations in asymmetric simple exclusion Prob. Theory Relat. Fields 91 81-101

Ferrari P A, Kipnis C and Saada E 1991 Microscopic structure of travelling waves for asymmetric simple exclusion process Ann. Prob. 19 226-44

Gradshteyn I S and Ryzhik I M 1980 Table of Integrals, Series and Products (London: Academic)

Gwa L H and Spohn H 1992 Six-vertex model, roughened surfaces and an asymmetric spin Hamiltonian Phys. Rev. Leth 68725 
Hakim V and Nadal J P 1983 Exact results for 2D directed animals on a strip of finite width J. Phys. A: Math. Gen. 16 L213

Janowsky S A and Lebowitz J L 1992 Finite-size effects and shock fiuctuations in the asymmetric simpleexclusion process Phys. Rev. A 45618

Jean-Marie A and Massey W A 1992 Steady state analysis for a series queueing network with blocking Preprint AT\& T Bell

Kandel D and Mukamel D 1992 Defects, interface profile and phase transitions in growth models Europhys. Leth 20325

Katz S, Lebowitz J L and Spohn H 1984 Nonequilibrium steady states of stochastic lattice gas models of fast ionic conductors J. Stat. Phys. 34497

Kipnis C 1986 Central limit theorems for infinite series of queues and applications to simple exclusion Ann. Prob. 14 397-408

Kipnis C, Lebowitz J L, Presutti E and Spohn H 1983 Self-diffusion of particles with stochastic collisions in one dimension $J$. Stat Phys. 30 107-22

Klümper A, Schadschneider A and Zittartz J 1991 Equivalence and solution of anisotropic spin-1 models and generalised $t-J$ fermion models in one dimension $J$. Phys. A: Math. Gen. 24 L955

Krug J 1991 Boundary-induced phase transitions in driven diffusive systems Phys. Rev. Leth 671882

Krug J and Spohn H 1991 Kinetic roughening of growing surfaces Solids far from Equilibrium ed C Godrèche (Cambridge: Cambridge University Press)

Liggett T M 1975 Ergodic theorems for the asymmetric simple exclusion process Trans. Am Math. Soc. 213237

1977 Ergodic theorems for the asymmetric simple exclusion process II Ann. Prob. 5 795-801 1985 Interacting Particle Systems (New York: Springer)

Meakin P, Ramanlal P, Sander L M and Ball R C 1986 Ballistic deposition on surfaces Phys. Rev. A 34 5091

Richards P M 1977 Theory of one-dimensional hopping conductivity and diffusion Phys. Rev. B 161393

Schütz $G 1992$ Generalized Bethe ansatz solution of a one-dimensional asymmetric exclusion process on a ring with blockage Preprint Weizmann Institute

Spitzer F 1970 Interaction of Markov Processes Adv. Math. 5 246-90

Spohn H 1991 Large Scale Dynamics of Interacting Particles (Berlin: Springer)

Sutherland B 1970 Two-dimensional hydrogen bonded crystals without the ice rule J. Math. Phys. 113183

van Beijeren H, Kehr K W and Kutner R 1983 Diffusion in concentrated lattice gases III. Tracer diffusion on a one-dimensional lattice Phys. Rev. B 285711 